\title{
Bistable Helmholtz bright solitons in saturable materials
}

\author{
J. M. Christian and G. S. McDonald \\ Joule Physics Laboratory, School of Computing, Science and Engineering, Institute for \\ Materials Research, University of Salford, Salford M5 4WT, United Kingdom

\section{P. Chamorro-Posada} \\ Departamento de Teoría de la Señal y Comunicaciones e Ingeniería Telemática, Universidad \\ de Valladolid, ETSI Telecomunicación, Campus Miguel Delibes s/n, \\ 47011 Valladolid, Spain.
}

\section{Submitted to J. Opt. Soc. Am. B}

\section{ABSTRACT}

We present, to the best of our knowledge, the first exact analytical solitons of a nonlinear Helmholtz equation with a saturable refractive-index model. These new two-dimensional spatial solitons have a bistable characteristic in some parameter regimes, and they capture oblique (arbitrary-angle) beam propagation in both the forward and backward directions. New conservation laws are reported, and the classic paraxial solution is recovered in an appropriate multiple limit. Analysis and simulations examine the stability of both solution branches, and stationary Helmholtz solitons are found to emerge from a range of perturbed input beams.

OCIS codes: 190.4400 (Nonlinear optics, materials), 190.4420 (Nonlinear optics, transverse effects in), 190.4390 (Nonlinear optics, integrated optics), 190.6135 (Spatial solitons), 190.3270 (Kerr effect), 190.5940 (Self-action effects). 


\section{INTRODUCTION}

Spatial solitons are robust self-localized optical beams that can evolve with a stationary intensity profile when diffractive spreading is exactly opposed by medium nonlinearity. In two-dimensional (2D) planar waveguides, where there is a longitudinal direction and effectively a single transverse direction, beams can be self-stabilizing. This innate stability against perturbations makes 2D spatial solitons ideal candidates for "building blocks" in future photonic devices [1-3].

Nonlinear Helmholtz (NLH) equations play a fundamental role in modelling many photonic systems. By respecting the inherent spatial symmetry of $2 \mathrm{D}$ uniform planar waveguides, many experimental configurations can be modelled that are otherwise inaccessible in conventional (paraxial) approaches. One key arena where Helmholtz models find unique applicability is in the accurate description of broad scalar beams in non-trivial angular geometries. Off-axis and oblique-incidence effects are recurrent themes in optics. For instance, even the most elementary arrangements - such as a pair of overlapping beams [4] or a beam impinging on the boundary between two materials [5] - are intrinsically angular scenarios. While paraxial analyses [6-9] are instructive and often yield invaluable results, they are valid strictly when the angles involved are nearly negligible (with respect to the reference direction).

In this paper, we consider wave propagation in materials with a saturable refractive index. Pivotal early works in the context of nonlinear guided waves were by Mihalache and coworkers [10-13], some two decades ago. The general phenomenon of saturation is important in many branches of nonlinear science, including beam/pulse propagation and nonparaxial optics, vortices and soliton clusters, spontaneous pattern formation, BoseEinstein condensates, and quantum-optical "squeezing."

Many materials are known to exhibit saturation, such as light-induced changes to their dispersive properties becoming bleached under high-intensity illumination. Examples include semiconductor-doped glasses (e.g., CdSSe and Schott OG 550 glass) [14,15], ion- 
doped crystals (e.g., $\mathrm{GdAlO}_{3}: \mathrm{Cr}^{3+}$ ) [16], bio-optical media [17], $\pi$-conjugated polymers [18] and various photorefractive crystals (e.g., $\mathrm{LiNbO}_{3}$ and $\mathrm{SBN}$ ) $[19,20]$. While the microphysics underlying saturation mechanisms is often complex, many of these materials have been described by simple models such as $n_{N L}(I) \sim I /\left(1+I / I_{\text {sat }}\right)$, where $n_{N L}$ is the nonlinear refractive index, $I$ is the local beam intensity, and $I_{\text {sat }}$ is a (material-dependent) saturation parameter [21].

Propagation equations with saturable nonlinearities tend not to possess exact analytical solutions $[22,23]$. This difficulty compels the theorist to look for other models that retain the essence of saturation, but that may instead permit exact analytical solutions to be found. The motivation is that exact solutions yield far more physical insight than computer simulations alone. Furthermore, the stability properties of solitons in saturable materials might reasonably be expected to be largely independent of the precise details of the function $n_{N L}(I)$

Phenomenological models of saturation often involve exponential- or polynomialtype intensity dependences [24]. However, here we are interested in the form proposed by Wood et al. [25],

$$
n_{N L}(I)=\frac{n_{2} I_{\mathrm{sat}}}{2}\left[1-\frac{1}{\left(1+I / I_{\mathrm{sat}}\right)^{2}}\right]
$$

At low intensities $n_{N L}(I) \sim n_{2} I$ (i.e., a Kerr-type nonlinearity) while at high intensities, $n_{N L}(I)$ flattens out to the constant value $n_{2} I_{\text {sat }} / 2$. Over fifteen years ago, Krolikowski and Luther-Davies [26] derived an exact analytical bright solition solution for a paraxial governing equation with nonlinearity (1). Their classic solution has a bistable characteristic: there exist pairs of beams with the same full-width-at-half-maximum (FWHM) but different peak intensities (and thus different integrated powers). This type of non-degenerate bistability, introduced by Gatz and Herrmann [23,27], differs from Kaplan's degenerate bistability $[28,29]$ (where beams with different propagation constants may possess the same power). 
Here, we present the first exact analytical solitons for an NLH equation with the saturable-nonlinearity model given in Eq. (1). These solutions are unconstrained by the slowly-varying envelope approximation, and describe optical propagation in generic saturable materials. Crucially, evolution may occur at any angle with respect to the reference direction $[4,5,30]$. These new solutions also retain a bistability characteristic, in common with their paraxial counterpart [26].

The layout of this paper is as follows. In Section 2, we propose a Helmholtz governing equation. Two novel families of exact analytical bright soliton solution, describing forward and backward beams, are derived. Their geometrical and bistability properties are discussed, and new conservation laws are presented. We also detail the recovery of the Krolikowski-Luther-Davies solution. In Section 3, computer simulations examine the stability of the new solitons against arbitrarily-large perturbations to the beam shape. We conclude, in Section 4, with some remarks about the potential applications of our work.

\section{HELMHOLTZ SOLITONS}

\section{A. Ultranarrow-Beam Self-Focusing}

Throughout this paper, we consider a beam whose waist $w_{0}$ is much broader than its freespace wavelength $\lambda$. When $\varepsilon \equiv \lambda / w_{0} \ll O(1)$, waves have a purely transverse character and can be treated within the scalar approximation. Order-of-magnitude corrections [31-34] (in successive powers of $\left.\varepsilon^{2}\right)$ from the polarization-scrambling term $\nabla(\nabla \cdot \mathbf{E})$ in Maxwell's equations are thus unimportant. When these contributions are negligible for an on-axis beam, they must also be negligible for the same beam evolving off-axis at angle $\theta$. Rotational invariance demands that the physical nature of any beam cannot depend on the relative orientation of the observer's coordinate axes; NLH models respect this fundamental symmetry. 


\section{B. Field and Envelope Equations}

We consider a transverse-electric (TE) polarized continuous-wave scalar electric field $\tilde{E}(x, z, t)=E(x, z) \exp (-i \omega t)+$ c.c. with angular frequency $\omega$, and where $E(x, z)$ satisfies the Maxwell field equation [30],

$$
\left(\frac{\partial^{2}}{\partial z^{2}}+\frac{\partial^{2}}{\partial x^{2}}\right) E(x, z)+\frac{\omega^{2} n^{2}}{c^{2}} E(x, z)=0 .
$$

Spatial symmetry is manifest in Eq. (2) as invariance under the permutation $x \leftrightarrow z$, and diffraction is fully-2D (occurring in both $x$ and $z$ ). Explicit $x-z$ equivalence permits waves to propagate and interact at arbitrary angles (relative to the reference direction ${ }^{4}$ ) and orientations (with respect to each other).

The total refractive index is $n=n_{0}+n_{N L}(I)$, where $n_{0}$ is the linear index, $n_{N L}(I)$ is the intensity-dependent contribution [given in Eq. (1)], and $I \equiv|E|^{2}$. For a weak nonlinearity, we have $n^{2} \simeq n_{0}^{2}+2 n_{0} n_{N L}(I)$. By choosing the $z$ axis as the longitudinal direction and transforming to the forward reference frame by introducing $E(x, z)=E_{0} u(x, z) \exp (i k z)$, one may derive an equation for the dimensionless envelope $u(x, z)$ without further approximation:

$$
\kappa \frac{\partial^{2} u}{\partial \zeta^{2}}+i \frac{\partial u}{\partial \zeta}+\frac{1}{2} \frac{\partial^{2} u}{\partial \xi^{2}}+\frac{1}{2} \frac{2+\gamma|u|^{2}}{\left(1+\gamma|u|^{2}\right)^{2}}|u|^{2} u=0
$$

Here, $\zeta=z / L_{D}$ and $\xi=2^{1 / 2} x / w_{0}$ are the normalized longitudinal and transverse coordinates, respectively, where $L_{D}=k w_{0}^{2} / 2$ is the diffraction length of a reference Gaussian beam. The inverse beam width is quantified by $\kappa=1 /\left(k w_{0}\right)^{2}=\varepsilon^{2} / 4 \pi^{2} n_{0}^{2} \ll O(1)$, where $k=n_{0} k_{0}$, and $k_{0}=\omega / c=2 \pi / \lambda$. Finally, $E_{0} \equiv\left(n_{0} / n_{2} k L_{D}\right)^{1 / 2}$ and $1 / \gamma \equiv I_{\text {sat }} / E_{0}^{2}$ is the normalized saturation intensity. The full generality of the in-plane Laplacian $\nabla^{2}=\partial_{z z}+\partial_{x x}$ has been preserved in both models (2) and (3). 


\section{Exact Analytical Solutions}

We seek exact analytical solutions to Eq. (3) that have the form $u(\xi, \zeta)=\rho^{1 / 2}(\xi, \zeta) \exp \left[i\left(K_{\xi} \xi+K_{\zeta} \zeta\right)\right] \exp (-i \zeta / 2 \kappa)$, where $\rho$ is the (real, positive) intensity distribution, $\mathbf{K} \equiv\left(K_{\xi}, K_{\zeta}\right)$ is the dimensionless wavevector, and $\exp (-i \zeta / 2 \kappa) \equiv \exp (-i k z)$ is the rapid-phase term that appears explicitly in Helmholtz envelope solutions (due to the $\kappa \partial_{\zeta \zeta}$ operator). Substituting the expression for $u$ into Eq. (3) and collecting the real and imaginary parts, one obtains

$$
\frac{2}{\rho}\left(\frac{\partial^{2} \rho}{\partial \xi^{2}}+2 \kappa \frac{\partial^{2} \rho}{\partial \zeta^{2}}\right)-\frac{1}{\rho^{2}}\left[\left(\frac{\partial \rho}{\partial \xi}\right)^{2}+2 \kappa\left(\frac{\partial \rho}{\partial \zeta}\right)^{2}\right]=8 \beta-4 \rho \frac{2+\gamma \rho}{(1+\gamma \rho)^{2}}
$$

and

$$
2 \kappa K_{\zeta} \frac{1}{\rho} \frac{\partial \rho}{\partial \zeta}+K_{\xi} \frac{1}{\rho} \frac{\partial \rho}{\partial \xi}=0
$$

where

$$
\kappa K_{\zeta}^{2}-\frac{1}{4 \kappa}+\frac{1}{2} K_{\xi}^{2} \equiv \beta
$$

We note that Eq. (4c) describes an ellipse in the $\left(K_{\xi}, K_{\zeta}\right)$ plane. The mathematical significance of the parameter $\beta$ will be discussed shortly. It is now convenient to introduce the change-of-variables $s \equiv(\xi+V \zeta)\left(1+2 \kappa V^{2}\right)^{-1 / 2}$, where $s$ is the coordinate perpendicular to the beam's propagation direction [35] and $-\infty \leq V \leq+\infty$ is the conventional transverse velocity parameterizing the rotation in the $(\xi, \zeta)$ plane. The operators $\partial_{\xi}$ and $\partial_{\zeta}$ transform as $\partial_{\xi}=\left(1+2 \kappa V^{2}\right)^{-1 / 2} d_{s}$ and $\partial_{\zeta}=V\left(1+2 \kappa V^{2}\right)^{-1 / 2} d_{s}$ so that Eq. (4a) becomes the quadrature equation

$$
\frac{d}{d \rho}\left[\frac{1}{\rho}\left(\frac{d \rho}{d s}\right)^{2}\right]=8 \beta-4 \frac{\rho(2+\gamma \rho)}{(1+\gamma \rho)^{2}}
$$


while Eq. (4b) uncovers the relationship $K_{\xi}=-2 \kappa V K_{\zeta}$. By using this expression to eliminate $K_{\xi}$ from Eq. (4c), one obtains two possible solutions for $K_{\zeta}$. Thus, the soliton wavevector is given by

$$
\mathbf{K} \equiv\left(K_{\xi}, K_{\zeta}\right)= \pm \sqrt{\frac{1+4 \kappa \beta}{1+2 \kappa V^{2}}}\left(-V,+\frac{1}{2 \kappa}\right)
$$

The beam is oriented along the line $\xi+V \zeta=0$ in the $(\xi, \zeta)$ plane, and its propagation direction (relative to the $+z$ direction) is determined by the choice of sign in $\mathbf{K}$. The \pm sign denotes evolution that is forward/backward in space, respectively (see Fig. 1).

To complete the solution, Eq. (5) must be supplemented by the familiar bright-soliton boundary conditions: $\rho=\rho_{0}$ and $(1 / \rho)(d \rho / d s)^{2}=0$ at $s=0$ (beam centre); $\rho=0$ and $(1 / \rho)(d \rho / d s)^{2}=0$ as $|s| \rightarrow \infty$ (beam extremes). Integration of Eq. (5), subject to these conditions, yields [26]

$$
\left(\frac{d \rho}{d s}\right)^{2}=\frac{4}{1+\gamma \rho_{0}}\left(\frac{\rho_{0}-\rho}{1+\gamma \rho}\right) \rho^{2}
$$

where the boundary conditions allow one to identify

$$
\beta \equiv \beta\left(\rho_{0}, \gamma\right)=\frac{1}{1+\gamma \rho_{0}}\left(\frac{\rho_{0}}{2}\right) .
$$

Integration of Eq. (7) yields an implicit equation for the intensity profile $\rho$. Finally, one can express the soliton solutions to Eq. (3) as

$$
u(\xi, \zeta)=\rho^{1 / 2}(\xi, \zeta) \exp \left[ \pm i \sqrt{\frac{1+4 \kappa \beta}{1+2 \kappa V^{2}}}\left(-V \xi+\frac{\zeta}{2 \kappa}\right)\right] \exp \left(-i \frac{\zeta}{2 \kappa}\right)
$$

where

$$
2 \tan ^{-1}(\Psi)+\frac{1}{\sqrt{\gamma \rho_{0}}} \ln \left(\frac{\Psi+\sqrt{\gamma \rho_{0}}}{\Psi-\sqrt{\gamma \rho_{0}}}\right)=\frac{1}{\sqrt{\gamma}} \frac{2}{\sqrt{1+\gamma \rho_{0}}}\left(\frac{\xi+V \zeta}{\sqrt{1+2 \kappa V^{2}}}\right)
$$

and

$$
\Psi \equiv\left(\gamma \frac{\rho_{0}-\rho}{1+\gamma \rho_{0}}\right)^{1 / 2}
$$


The forward solution describes a beam propagating at angle $-90^{\circ} \leq \theta \leq+90^{\circ}$ relative to the reference (longitudinal) axis [see Fig. 2(a)]. The corresponding backward solution describes the same beam propagating in the opposite direction, which is equivalent to reversing the components of the wavevector (i.e., $\mathbf{K} \rightarrow-\mathbf{K}$ ).

The two solutions are related by a $180^{\circ}$ - rotation of either the beam itself or the observer's coordinate axes. In other words, there is only a single physical beam. Through the trigonometric relations $\cos \theta=\left(1+2 \kappa V^{2}\right)^{-1 / 2}$ and $\sin \theta=(2 \kappa)^{1 / 2} V\left(1+2 \kappa V^{2}\right)^{-1 / 2}$, one may eliminate $V$ and construct the symmetric solution

$$
u(\xi, \zeta)=\rho^{1 / 2}(\xi, \zeta) \exp \left[i \sqrt{\frac{1+4 \kappa \beta}{2 \kappa}}\left(-\xi \sin \theta+\frac{\zeta}{\sqrt{2 \kappa}} \cos \theta\right)\right] \exp \left(-i \frac{\zeta}{2 \kappa}\right),
$$

and

$$
2 \tan ^{-1}(\Psi)+\frac{1}{\sqrt{\gamma \rho_{0}}} \ln \left(\frac{\Psi+\sqrt{\gamma \rho_{0}}}{\Psi-\sqrt{\gamma \rho_{0}}}\right)=\frac{1}{\sqrt{\gamma}} \frac{2}{\sqrt{1+\gamma \rho_{0}}}\left(\xi \cos \theta+\frac{\zeta}{\sqrt{2 \kappa}} \sin \theta\right)
$$

where the propagation angle now lies within the range $-180^{\circ} \leq \theta \leq+180^{\circ}$.

\section{Soliton Geometry}

Equations (9) and (10) are exact analytical solutions to the governing equation (3). Generic features, i.e., $\kappa, \kappa \beta$, and $\kappa V^{2}$ contributions, appearing in the beam phase arise from the Helmholtz term $\kappa \partial_{\zeta \zeta}$. These features have no analogue in paraxial modelling.

A potentially-dominant Helmholtz effect is the factor $\left(1+2 \kappa V^{2}\right)^{1 / 2}=\sec \theta$ in Eq. (9b). For example, consider the moderate angle $|\theta|=60^{\circ}$. Since $\tan ^{2} \theta \equiv 2 \kappa V^{2}=3$, the beam width, as perceived by an observer in the $(x, z)$ frame, is doubled relative to its on-axis value (see Fig. 3). This is a $100 \%$ correction to the prediction of paraxial theory [26]. Similarly, when $|\theta| \rightarrow 90^{\circ}$, the beam appears to be infinitely wide since evolution takes place perpendicularly to the reference direction. Propagation angles of such magnitude are clearly inaccessible to paraxial theory. 
Angular beam broadening is a purely geometrical effect whose contribution can be of any order, independently of $\kappa$. Geometrical broadening, in turn, becomes critical in understanding the oblique refraction [5] or interactions [4] of such beams. Its accurate description requires the full presence of $\partial_{z z}$ in the governing equation. Perturbative corrections to the envelope equation [31-34] (e.g., expansions involving higher-order powers of the transverse-diffraction operator to approximate $\kappa \partial_{\zeta \zeta}$ ) are, in general, not sufficiently flexible to capture arbitrary-angle effects.

\section{E. Non-Degenerate Bistability}

In the solution continuum, one can identify pairs of beams that have the same FWHM but different peak intensities [26]. In defining a half-width condition $\rho(s=v \Delta)=\rho_{0} / 2$, where $\Delta \equiv \operatorname{sech}^{-1}\left(2^{-1 / 2}\right) \simeq 0.8814, v$ represents the beam half-width in units of $\Delta$. For example, $v=1$ defines canonical solutions that have a half-width-at-half-maximum of $s=\Delta$, and thus a FWHM of $2 \Delta$. Substituting the half-width condition into solution (9), it can be shown that

$$
2 \tan ^{-1}\left[\left(\frac{\gamma \rho_{0}}{2+\gamma \rho_{0}}\right)^{1 / 2}\right]+\frac{1}{\sqrt{\gamma \rho_{0}}} \ln \left(\frac{\sqrt{2+\gamma \rho_{0}}+1}{\sqrt{2+\gamma \rho_{0}}-1}\right)=\frac{2 v \Delta}{\sqrt{\gamma} \sqrt{1+\gamma \rho_{0}}} .
$$

Typical non-degenerate bistability curves are shown in Fig. 4. When the saturation parameter $\gamma$ is less than a critical value, i.e., $\gamma<\gamma_{\text {crit }}$, there are two values of $\rho_{0}$ that satisfy Eq. (11). As $v$ increases (i.e., the FWHM of the beam becomes larger), one finds that the bistable region extends over a broader range of $\gamma$ (i.e., $\gamma_{\text {crit }}$ increases).

Equation (11) provides a relationship between the saturation parameter $\gamma$ and the peak intensity $\rho_{0}$ for a beam with a FWHM of $2 v \Delta$. Since the FWHM is defined in the direction perpendicular to the propagation axis, the values of $\rho_{0}$ that satisfy Eq. (11) must be independent of $\theta$. This manifestation of spatial symmetry was uncovered for the recentlyderived bistable solitons of a cubic-quintic Helmholtz equation [36]. 
It is instructive to consider the limits associated with the lower and upper solution branches. As $\gamma \rho_{0} \rightarrow 0$ [i.e., $I / I_{\text {sat }} \ll O(1)$ in unscaled units], the lower branch follows $\rho_{0} \sim 1 / v^{2}$ (this result coincides with that for a Kerr bright soliton, as expected). However, as $\gamma \rho_{0} \rightarrow \infty$, the upper-branch intensity diverges as $\rho_{0} \sim \gamma^{-1}\left[\gamma^{-1}(4 v \Delta / \pi)^{2}-1\right]$

\section{F. Conservation Laws}

Conserved quantities for Eq. (3) can be derived using a field-theoretic approach [37]. From the Lagrangian density $\mathcal{L}$, where

$$
\mathcal{L}=\frac{i}{2}\left(u^{*} \frac{\partial u}{\partial \zeta}-u \frac{\partial u^{*}}{\partial \zeta}\right)-\kappa \frac{\partial u^{*}}{\partial \zeta} \frac{\partial u}{\partial \zeta}-\frac{1}{2} \frac{\partial u^{*}}{\partial \xi} \frac{\partial u}{\partial \xi}+G\left(u, u^{*}\right)
$$

and

$$
G\left(u, u^{*}\right) \equiv \int_{0}^{u^{*} u} d Y \frac{1}{2} \frac{(2+\gamma Y) Y}{(1+\gamma Y)^{2}}=\frac{1}{2} \frac{\left(u^{*} u\right)^{2}}{\left(1+\gamma u^{*} u\right)}
$$

one can define a pair of canonically-conjugate momentum variables, denoted by $\pi$ and $\tilde{\pi}$, respectively:

$$
\pi \equiv \frac{\partial \mathcal{L}}{\partial u_{\zeta}}=\left(\frac{i}{2}-\kappa \frac{\partial}{\partial \zeta}\right) u^{*}, \quad \tilde{\pi} \equiv \frac{\partial \mathcal{L}}{\partial u_{\zeta}^{*}}=-\left(\frac{i}{2}+\kappa \frac{\partial}{\partial \zeta}\right) u,
$$

where $u_{\zeta} \equiv \partial u / \partial \zeta$, etc. The Euler-Lagrange equations $\not \not_{L} / \not \not_{u}{ }^{*}=0$ and $\not \not_{L} / \not \partial_{u}=0$, where $\not \supset / \not u^{\prime}$ and $\not \supset / \not u_{u}$ denote variational derivatives, then give rise to Eq. (3) and its complex conjugate, respectively. By considering the invariance of $\mathcal{L}$ under a set of continuous oneparameter transformations [37] (a global phase change, and infinitesimal translations in $\xi$ and $\zeta$ ), one can arrive at the three integral conserved quantities:

$$
\begin{gathered}
W=\int_{-\infty}^{+\infty} d \xi\left[|u|^{2}-i \kappa\left(u^{*} \frac{\partial u}{\partial \zeta}-u \frac{\partial u^{*}}{\partial \zeta}\right)\right], \\
M=\int_{-\infty}^{+\infty} d \xi\left[\frac{i}{2}\left(u^{*} \frac{\partial u}{\partial \xi}-u \frac{\partial u^{*}}{\partial \xi}\right)-\kappa\left(\frac{\partial u^{*}}{\partial \xi} \frac{\partial u}{\partial \zeta}+\frac{\partial u^{*}}{\partial \zeta} \frac{\partial u}{\partial \xi}\right)\right],
\end{gathered}
$$


and

$$
H=\int_{-\infty}^{+\infty} d \xi\left[\frac{1}{2} \frac{\partial u^{*}}{\partial \xi} \frac{\partial u}{\partial \xi}-\kappa \frac{\partial u^{*}}{\partial \zeta} \frac{\partial u}{\partial \zeta}-G\left(u, u^{*}\right)\right]
$$

These expressions for the energy-flow, momentum, and Hamiltonian, respectively, hold for both forward and backward solutions. Aside from their physical and mathematical significance, the conserved quantities are particularly important for tracking the accuracy of the algorithm used to integrate Eq. (3) numerically [38].

By substituting $u(\xi, \zeta)=\rho^{1 / 2}(\xi, \zeta) \exp \left[i\left(K_{\xi} \xi+K_{\zeta} \zeta\right)\right] \exp (-i \zeta / 2 \kappa)$ into Eqs. (14a)-(14c), one can simplify the conserved integral quantities to a set of compact algebraic relations:

$$
\begin{gathered}
W= \pm(1+4 \kappa \beta)^{1 / 2} P, \\
M=\frac{V}{\sqrt{1+2 \kappa V^{2}}}[(1+4 \kappa \beta) P-2 \kappa Q], \\
H=\frac{W}{2 \kappa}-\frac{1}{\sqrt{1+2 \kappa V^{2}}}\left(\frac{1}{2 \kappa}\right)[(1+4 \kappa \beta) P-2 \kappa Q],
\end{gathered}
$$

where $P \equiv P(\beta, \gamma)$ and $Q \equiv Q(\beta, \gamma)$ are given by the integrals

$$
P \equiv \int_{-\infty}^{+\infty} d s \rho(s)
$$

and

$$
Q \equiv \frac{1}{4} \int_{-\infty}^{+\infty} d s \frac{1}{\rho(s)}\left[\frac{d \rho(s)}{d s}\right]^{2} .
$$

It is interesting to note that Helmholtz bright solitons satisfy the classical-particle energymomentum relationship $\partial H / \partial M=\partial_{V} H / \partial_{V} M=V$.

\section{G. The Paraxial Limit}

While it is tempting to expect recovery of the Krolikowski-Luther-Davies paraxial soliton [26] simply by setting $\kappa \simeq 0$, this is actually untrue. The limit process is much more subtle 
(both physically and mathematically), and one must ensure that all contributions from the Helmholtz term $\kappa \partial_{\zeta \zeta}$ are negligible simultaneously. This requires $\kappa \rightarrow 0$ (broad beams), $\kappa \beta \rightarrow 0$ (moderate intensities), and $\kappa V^{2} \rightarrow 0$ (negligible propagation angles). From the forward solution, we obtain,

$$
u(\xi, \zeta) \sim \rho^{1 / 2}(\xi, \zeta) \exp \left[-i V \xi+i\left(\beta-\frac{V^{2}}{2}\right) \zeta\right]
$$

and

$$
2 \tan ^{-1}(\Psi)+\frac{1}{\sqrt{\gamma \rho_{0}}} \ln \left(\frac{\Psi+\sqrt{\gamma \rho_{0}}}{\Psi-\sqrt{\gamma \rho_{0}}}\right) \sim \frac{2}{\sqrt{\gamma}} \frac{\xi+V \zeta}{\sqrt{1+\gamma \rho_{0}}},
$$

as should be the case. From Eq. (16a), it is clear that $\beta$ [as defined in Eqs. (4c) and (8)] can be identified with the propagation constant of the corresponding on-axis paraxial beam. In the same way, one can also recover the paraxial conservation laws from Eqs. (15a)-(15c), namely $W \sim P, M \sim V P$, and $H \sim \frac{1}{2} V^{2} P-\beta P+Q$.

When applying the paraxial limit to the backward beam, where propagation is nearly on-axis but in the $-z$ direction, one finds that

$$
u(\xi, \zeta) \simeq \rho^{1 / 2}(\xi, \zeta) \exp \left[i V \xi-i\left(\beta-\frac{V^{2}}{2}\right) \zeta\right] \exp \left(-i 2 \frac{\zeta}{2 \kappa}\right)
$$

Similarly, the conserved quantities for the backward beam tend to $W \sim-P, M \sim V P$, and $H \sim \frac{1}{2} V^{2} P-3 \beta P+Q-P / \kappa$. Negative energy-flows do not appear in paraxial theory [since the integrand in Eq. (15d) is always positive-definite], and the Hamiltonian diverges as $\kappa^{-1}$. The corresponding paraxial model has no analogue of these results, which retain $\kappa$ dependent contributions. This emphasizes the fact that paraxial models support wave propagation in a single longitudinal direction only. 


\section{SOLITON STABILITY}

Recently, we addressed the linear stability of plane-wave solutions to generic NLH equations against small fluctuations $[39,40]$. Here, we investigate the robustness of the new bistable soliton (9) against localized perturbations (to the beam shape) that may be arbitrarily large. For this task, full numerical computations are essential.

\section{A. Stability Criterion}

Previously, the stability of Helmholtz bright solitons has been analysed by combining the Vakhitov-Kolokolov (VK) integral criterion [41] with spatial symmetry [36,42]. The VK inequality predicts that solitons of nonlinear Schrödinger-type equations can be robust against perturbations when

$$
\frac{d P}{d \beta}=\frac{d}{d \beta} \int_{-\infty}^{+\infty} d \xi|u(\xi, \zeta ; \beta)|^{2}>0
$$

From Kaplan's analysis [28,29], the curve $P(\beta)$ can be obtained implicitly from

$$
P(\beta)=\frac{1}{\sqrt{2}} \int_{0}^{\rho_{0}(\beta)} d \rho_{0}^{\prime}\left[\beta-\frac{1}{1+\gamma \rho_{0}^{\prime}}\left(\frac{\rho_{0}^{\prime}}{2}\right)\right]^{-1 / 2} .
$$

The upper limit on the integral is $\rho_{0}(\beta)=2 \beta /(1-2 \beta \gamma)$, which is consistent with Eq. (8). Since $0 \leq \rho_{0} \leq \infty$, the paraxial propagation constant $\beta$ must satisfy the inequality $0 \leq \beta \leq \beta_{\max }$, where $\beta_{\max } \equiv 1 / 2 \gamma$. In the limit $\gamma \rho_{0}^{\prime} \rightarrow 0$, one recovers from Eq. (19) the familiar result for Kerr solitons, i.e., $P=2(2 \beta)^{1 / 2}$.

On-axis $\left(V=0, \kappa V^{2}=0\right)$ forward-propagating Helmholtz beams with $\kappa \ll O(1)$ and $\kappa \beta \ll O(1)$ are identical to their paraxial counterparts [26], except for a negligibly-small correction to the phase shift [at $O(\kappa \beta)$ ]. Thus, if the paraxial soliton satisfies Eq. (18), then the Helmholtz generalization must also be stable. In uniform media, beam stability must be insensitive to arbitrary rotations of the observer's coordinate axes. Thus, spatial symmetry can be used to infer that if the on-axis beam is stable, then the same beam in an off-axis 
configuration must also be stable. Plots of $P(\beta)$ for four different values of the saturation parameter $\gamma$ are shown in Fig. 5. Since the slope is always positive, we expect the underlying Helmholtz solitons can be robust.

\section{B. Perturbed Input Beams}

The stability of Helmholtz solitons is now investigated through computer simulations. From the plethora of possible input beams, we restrict our attention to

$$
u(\xi, 0)=\rho^{1 / 2}(\xi, 0) \exp \left(-i V \sqrt{\frac{1+4 \kappa \beta}{1+2 \kappa V^{2}}} \xi\right),
$$

where $\rho(\xi, 0)$ is obtained from the paraxial relation, Eq. (16b). The perturbation thus arises

from omission of the characteristic Helmholtz broadening factor $\left(1+2 \kappa V^{2}\right)^{1 / 2}=\sec \theta$ from the soliton profile. Geometrically, this class of initial-value problem effectively addresses what happens when paraxial solutions are fed into inherently off-axis nonparaxial regimes [35]. For definiteness, we present specific results for launching angles of $|\theta|=10^{\circ}, 30^{\circ}$, and $50^{\circ}$. When $\kappa=10^{-3} \quad\left(\kappa=10^{-4}\right)$, these angles correspond to the transverse velocities $|V| \simeq 3.94,12.91$, and $26.65(|V| \simeq 12.47,40.82$, and 84.27), respectively.

\section{Stability of Canonical Solitons}

For brevity, results are presented for canonical beams (i.e., those with $v=1$ ). The saturation parameter is chosen to be $\gamma=0.25$, and the lower- and upper-branch solutions have $\rho_{0} \simeq 2.32$ and $\rho_{0} \simeq 8.68$, respectively [obtained by solving Eq. (11)]. Unperturbed solitons propagate with stationary profiles, as expected, providing numerical confirmation our expectations discussed in subsection 3A. Perturbed beams, on the other hand, tend to exhibit self-reshaping oscillations in their parameters (i.e., amplitude, width, and area $=$ amplitude $\times$ width - see Fig. 6), and shed a small amount of radiation in the process. 
The character of the reshaping oscillations depends upon the branch on which the launched soliton sits. A perturbed lower-branch beam tends to evolve asymptotically into an exact Helmholtz soliton. That is, as $\zeta \rightarrow+\infty$, the oscillations disappear and the beam approaches a stationary profile. However, perturbed upper-branch beams tend to exhibit sustained oscillations that do not appear to vanish as $\zeta \rightarrow+\infty$. By interpreting radiative losses as an internal mechanism for energy dissipation, one can classify the lower-branch solitons in Fig. 6 as stable fixed-point attractors, and upper-branch solitons as stable limitcycle attractors in the $\left(\rho_{0}, d \rho_{0} / d \zeta\right)$ phase plane $[36,39,42]$.

Figure 4 shows that when $v$ becomes larger, the peak intensity of the lower- (upper-) solution branch tends to decrease (increase). Comparing the oscillation periods in the two parts of Fig. 6, one might expect that as the beam FWHM increases, perturbed solitons lying on the lower (upper-) branch should reshape with characteristic oscillations that occur on a longer (shorter) scalelength. This prediction has been confirmed through simulations.

\section{CONCLUSIONS}

We have derived the first exact analytical Helmholtz solitons for a saturable nonlinearity. Complementary forward- and backward-travelling solutions have been combined into a single beam that can propagate at any angle relative to the reference (e.g., z) direction. Analysis has uncovered a bistable characteristic in certain parameter regimes, where the governing equation supports coexisting Helmholtz solitons with different peak intensities but identical FWHM. New conservation laws have also been presented, and the recovery of paraxial results has been discussed in detail.

Rigorous numerical simulations have shown that saturable Helmholtz solitons are generally stable against perturbations to their shape, and that they can be interpreted as robust attractors $[36,39,42]$ of the system. Since the nonlinearity studied here is generic, we expect that the stability properties of Helmholtz solitons in other saturation models $[24,25]$ (where exact analytical solutions may not necessarily exist) to be largely unchanged. 
The new solitons have intrinsic mathematical interest as exact solutions to spatiallysymmetric non-integrable partial differential equations. They provide the basis for understanding saturable solitons in novel angular geometries. Typical applications where one could deploy nonlinear-Helmholtz analyses [4,5] include spatial-soliton logic [43], dragging [44], switching [45,46], and computing with solitons [47]. There are also the possibilities of Helmholtz-type generalizations of bistable multibeam contexts [48], and new angular scenarios involving waveguide arrays [49]. Central to all these applications is a thorough understanding of the properties of the underlying Helmholtz solitons.

Helmholtz equations in general [50-53] offer a wealth of exciting possibilities, and will inevitably lead to many new avenues of research in the physics, mathematics, and computational analyses of nonlinear wave phenomena. We fully expect that such modelling will provide a key analytical platform for a wide range of future applications exploiting optical fields beyond the paraxial approximation. 


\section{REFERENCES}

[1] G. I. Stegeman and M. Segev, Science 286, "Optical spatial solitons and their interactions: universality and diversity," 1518-1523 (1999).

[2] Y. S. Kivshar, "Bright and dark spatial solitons in non-Kerr media," Opt. Quantum Electron. 30, 571-614 (1998).

[3] Y. S. Kivshar and B. Luther-Davies, "Dark optical solitons: physics and applications," Phys. Rep. 298, 81-197 (1998).

[4] P. Chamorro-Posada and G. S. McDonald, "Spatial Kerr soliton collisions at arbitrary angles," Phys. Rev. E 74, 036609 (2006).

[5] J. Sánchez-Curto, P. Chamorro-Posada, and G. S. McDonald, "Helmholtz solitons at nonlinear interfaces," Opt. Lett. 32, 1126-1128 (2007).

[6] J. P. Gordon, "Interaction forces among solitons in optical fibers," Opt. Lett. 8, 596598 (1983).

[7] O. Cohen, R. Uzdin, T. Carmon, J. Fleischer, M. Segev, and S. Odouov, "Collisions between optical spatial solitons propagating in opposite directions," Phys. Rev. Lett. 89, $133901(2002)$.

[8] A. B. Aceves, J. V. Moloney, and A. C. Newell, "Theory of light-beam propagation at nonlinear interfaces. I. Equivalent-particle theory for a single interface,” Phys. Rev. A 39, 1809-1827 (1989).

[9] A. B. Aceves, J. V. Moloney, and A. C. Newell, "Theory of light-beam propagation at nonlinear interfaces. II. Multiple-particle and multiple-interface extensions,” Phys. Rev. A 39, 1828-1840 (1989).

[10] D. Mihalache and D. Mazilu, "TM-polarized nonlinear slab-guided waves in saturable media," Solid State Commun. 60, 397-399 (1986).

[11] D. Mihalache and D. Mazilu, "Stability and instability of nonlinear guided waves in saturable media," Solid State Commun. 63, 215-217 (1987). 
[12] D. Mihalache and D. Mazilu, "Stability of nonlinear slab-guided waves in saturable media: a numerical analysis," Phys. Lett. A 122, 381-384 (1987).

[13] D. Mihalache, D. Mazilu, M. Bertolotti, and C. Sibilia, "Exact solution for transverse electric polarized nonlinear guided waves in saturable media,” J. Mod. Opt. 35, 10171027 (1988).

[14] P. Roussignol, D. Ricard, J. Lukasik, and C. Flytzanis, "New results on optical phase conjugation in semiconductor-doped glasses," J. Opt. Soc. Am. B 4, 5-13 (1987).

[15] J.-L. Coutaz and M. Kull, "Saturation of the nonlinear index of refraction in semiconductor-doped glass,” J. Opt. Soc. Am. B 8, 95-98 (1991).

[16] T. Catunda and L. A. Cury, "Transverse self-phase modulation in ruby and $\mathrm{GdAlO}_{3}: \mathrm{Cr}^{+3}$ crystals," J. Opt. Soc. Am. B 7, 1445-1455 (1990).

[17] Q. Wang Song, X. Wang, R. R. Birge, J. D. Downie, D. Timucin, and C. Gary, "Propagation of a Gaussian beam in a bacteriorhodopsin film," J. Opt. Soc. Am. B 15, 1602-1609 (1998).

[18] L. Demenicis, A. S. L. Gomes, D. V. Petrov, C. B. de Araújo, C. P. de Melo, C. G. dos Santos, and R. Souto-Maior, "Saturation effects in the nonlinear-optical susceptibility of poly(3-hexadecylthiophene),” J. Opt. Soc. Am. B 14, 609-614 (1997).

[19] S. Bian, J. Frejlich, and K. H. Ringhofer, "Photorefractive saturable Kerr-type nonlinearity in photovoltaic crystals," Phys. Rev. Lett. 78, 4035-4038 (1997).

[20] D. N. Christodoulides and M. I. Carcalho, "Bright, dark, and grey spatial soliton states in photorefractive media,” J. Opt. Soc. Am. B 12, 1628-1633 (1995).

[21] S. Bian, M. Martinelli, and R. J. Horowicz, "Z-scan formula for saturable Kerr media," Opt. Commun. 172, 347-353 (1999).

[22] S. Gatz and J. Herrmann, "Soliton propagation in materials with saturable nonlinearity," J. Opt. Soc. Am. B 8, 2296-2302 (1991).

[23] J. Herrmann, "Propagation of ultrashort light pulses in fibers with saturable nonlinearity in the normal-dispersion region,” J. Opt. Soc. Am. B 8, 1507-1511 (1991). 
[24] D. Mihalache, M. Bertolotti, and C. Sibilia, "Nonlinear wave propagation in planar structures," Prog. Opt. 27, 229-313 (1989).

[25] V. E. Wood, E. D. Evans, and R. P. Kenan, "Soluble saturable refractive-index nonlinearity model,” Opt. Commun. 69, 156-160 (1988).

[26] W. Krolikowski and B. Luther-Davies, "Analytic solution for soliton propagation in a nonlinear saturable medium,” Opt. Lett. 17, 1414-1416 (1992).

[27] S. Gatz and J. Herrmann, "Soliton collision and soliton fusion in dispersive materials with a linear and quadratic intensity depending refraction index change," IEEE J. Quantum Electron. 28, 1732-1738 (1992).

[28] A. E. Kaplan, "Bistable solitons," Phys. Rev. Lett. 55, 1291-1294 (1985).

[29] A. E. Kaplan, "Multistable self-trapping of light and multistable soliton pulse propagation,” IEEE J. Quantum Electron. QE-21, 1538-1543 (1985).

[30] P. Chamorro-Posada, G. S. McDonald, and G. H. C. New, "Exact soliton solutions of the nonlinear Helmholtz equation: communication,” J. Opt. Soc. Am. B 19, 1216-1217 (2002).

[31] M. Lax, W. H. Louisell, and W. B. McKnight, "From Maxwell to paraxial wave optics,” Phys. Rev. A 11, 1365-1370 (1975).

[32] S. Chi and Q. Guo, "Vector theory of self-focusing of an optical beam in Kerr media," Opt. Lett. 20, 1598-1600 (1995).

[33] B. Crosignani, A. Yariv, and S. Mookherjea, "Nonparaxial spatial solitons and propagation-invariant pattern solutions in optical Kerr media," Opt. Lett. 29, 12541256 (2004).

[34] A. Ciattoni. B. Crosignani, S. Mookherjea, and A. Yariv, "Nonparaxial dark solitons in optical Kerr media,” Opt. Lett. 30, 516-518 (2005).

[35] P. Chamorro-Posada, G. S. McDonald, and G. H. C. New, "Propagation properties of nonparaxial spatial solitons,” J. Mod. Opt. 47, 1877-1886 (2000).

[36] J. M. Christian, G. S. McDonald, and P. Chamorro-Posada, "Bistable Helmholtz solitons in cubic-quintic materials," Phys. Rev. A 76, 033833 (2007). 
[37] H. Goldstein, Classical Mechanics, $2^{\text {nd }}$ Ed. (Addison-Wesley 1980).

[38] P. Chamorro-Posada, G. S. McDonald, and G. H. C. New, "Nonparaxial beam propagation methods," Opt. Commun. 192, 1-12 (2001).

[39] J. M. Christian, G. S. McDonald, and P. Chamorro-Posada, "Helmholtz bright and boundary solitons,” J. Phys. A: Math. Theor. 40, 1545-1560 (2007).

[40] J. M. Christian, G. S. McDonald, and P. Chamorro-Posada, "Helmholtz bright and boundary solitons," J. Phys. A: Math. Theor. Corrigendum 40, 8601 (2007).

[41] M. G. Vakhitov and A. A. Kolokolov, "Stationary solutions of the wave equation in the medium with nonlinearity saturation,” Radiophys. Quantum Electron. 16, 783-789 (1973).

[42] J. M. Christian, G. S. McDonald, R. J. Potton, and P. Chamorro-Posada, "Helmholtz solitons in power-law optical materials," Phys. Rev. A 76, 033834 (2007).

[43] S. Blair and K. Wagner, "Spatial soliton angular deflection logic gates," Appl. Opt. 38, 6749-6772 (1999).

[44] J. U. Kang, G. I. Stegeman, and J. S. Aitchison, "One-dimensional spatial soliton dragging, trapping, and all-optical switching in AlGaAs waveguides," Opt. Lett. 21, 189-191 (1996).

[45] Y.-D. Wu, "New all-optical switch based on the spatial soliton repulsion," Opt. Exp. 14, 4005-4012 (2006).

[46] Y.-D. Wu, "All-optical $1 \times N$ switching device by use of the phase modulation of spatial solitons," Appl. Opt. 44, 4144-4147 (2005).

[47] C. Anastassiou, M. Segev, K. Steiglitz, J. A. Giordmaine, M. Mitchell, M. Shih, S. Lan, and J. Martin, "Energy-exchange interactions between colliding vector solitons," Phys. Rev. Lett. 83, 2332-2335 (1999).

[48] J. Scheuer and M. Orenstein, "Interactions and switching of spatial soliton pairs in the vicinity of a nonlinear interface,” Opt. Lett. 24, 1735-1737 (1999).

[49] Y. V. Kartashov, A. S. Zelenina, L. Torner, and V. A. Vysloukh, "Spatial soliton switching in quasi-continuous optical arrays," Opt. Lett. 29, 766-768 (2004). 
[50] M. D. Feit and J. A. Fleck, "Beam nonparaxiality, filament formation, and beam breakup in the self-focusing of optical beams," J. Opt. Soc. Am. B 5, 633- 640 (1988).

[51] A. P. Sheppard and M. Haelterman, "Nonparaxiality stabilizes three dimensional soliton beams in Kerr media,” Opt. Lett. 23, 1820-1822 (1998).

[52] T. A. Laine and A. T. Friberg, "Self-guided waves and exact solutions of the nonlinear Helmholtz equation,” J. Opt. Soc. Am. B 17, 751-757 (2000).

[53] G. Baruch, G. Fibich, and S. Tsynkov, "High-order numerical solution of the nonlinear Helmholtz equation with axial symmetry,” J. Comp. Appl. Math. 204, 477-492 (2007). 


\section{FIGURE CAPTIONS}

Fig. 1. (Color online) Geometry of Helmholtz solitons in the $(\xi, \zeta)$ plane. The forward solutions have $K_{\zeta}>0$, while the direction of $K_{\xi}$ is defined by $V\left[\right.$ (a) $K_{\xi}<0$ when $V>0$; (b) $K_{\xi}>0$ when $\left.V<0\right]$. The backward solutions have $K_{\zeta}<0\left[\right.$ (c) $K_{\xi}>0$ when $V>0$; (d) $K_{\xi}<0$ when $\left.V<0\right]$. The red (dashed) line marks $\xi+V \zeta=0$, where $|V| \rightarrow \infty$ is when the beam coincides with the $\xi$ axis. The propagation angle of the beam with respect to the $\zeta$ axis is $\Theta$, where $\tan \Theta \equiv \partial K_{\zeta} / \partial K_{\xi}=\partial_{V} K_{\zeta} / \partial_{V} K_{\xi}=V$ and $\partial_{V} \equiv \partial / \partial V$.

Fig. 2. (Color online) Propagation domains for (a) forward and (b) backward Helmholtz solitons. Each beam is restricted by the condition $-\infty \leq V \leq+\infty$, which corresponds to $-90^{\circ} \leq \theta \leq+90^{\circ}$ in the $(x, z)$ frame since $\tan \theta=(2 \kappa)^{1 / 2} V$. The grey region denotes forbidden directions. Since $x$ and $z$ are scaled by different factors $\left(L_{D} \gg w_{0}\right)$, the propagation angle $\theta$ in the $(x, z)$ frame is represented by the angle $\Theta$ in the $(\xi, \zeta)$ frame (see Fig. 1), where $\theta$ and $\Theta$ are related by $\tan \theta=(2 \kappa)^{1 / 2} \tan \Theta$.

Fig. 3. (Color online) Angular beam-broadening effect for bistable Helmholtz solitons (9) when $\gamma=0.25$ for (a) lower- $\left(\rho_{0} \simeq 2.32\right)$ and (b) upper-branch $\left(\rho_{0} \simeq 8.68\right)$ solutions. Geometrical broadening is absent for the paraxial solution $\left(|\theta|=0^{\circ}\right)$. For a launching angle of $|\theta|=60^{\circ}$, the perceived width of the beam has doubled relative to the paraxial profile.

Fig. 4. (Color online) Curves defining non-degenerate bistable solution families for four different values of the width parameter $v$. These plots are obtained by solving Eq. (11) numerically. 
Fig. 5. (Color online) Paraxial beam power $P$ as a function of $\beta$ for four different values of the saturation parameter $\gamma$. The curve for $\gamma=0.00$ corresponds to a Kerr nonlinearity, where $\beta=\rho_{0} / 2$ [from Eq. (8)]. One finds that the slope $d P / d \beta$ is always positive (solitons predicted to be stable), and $P(\beta)$ is single-valued (no Kaplan-type degenerate bistability).

Fig. 6. (Color online) Beam reshaping simulations for (a) lower- and (b) upper-branch canonical solitons. Solid line: $|\theta|=10^{\circ}$, dashed line: $|\theta|=30^{\circ}$; dot-dashed line: $|\theta|=50^{\circ}$. Perturbed beams exhibit decaying oscillations in the amplitude, width, and area. These curves are universal, and hold for any combination of $\kappa V^{2}=\frac{1}{2} \tan ^{2} \theta$ [see text for the specific numerical values of $\kappa$ and $V$ used in these simulations]. 

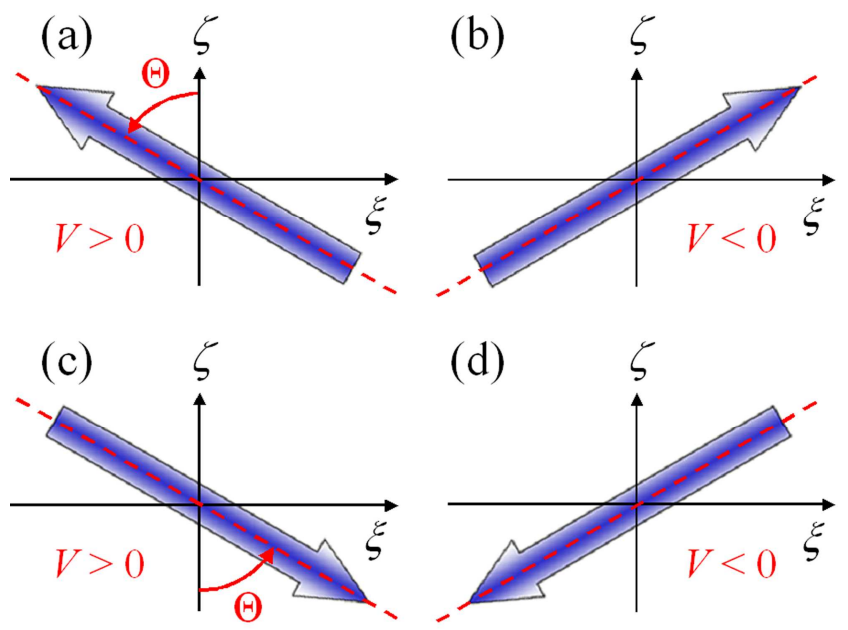

Fig. 1. (Color online) Geometry of Helmholtz solitons in the $(\xi, \zeta)$ plane. The forward solutions have $K_{\zeta}>0$, while the direction of $K_{\xi}$ is defined by $V\left[\right.$ (a) $K_{\xi}<0$ when $V>0$; (b) $K_{\xi}>0$ when $V<0$ ]. The backward solutions have $K_{\zeta}<0$ [(c) $K_{\xi}>0$ when $V>0$; (d) $K_{\xi}<0$ when $\left.V<0\right]$. The red (dashed) line marks $\xi+V \zeta=0$, where $|V| \rightarrow \infty$ is when the beam coincides with the $\xi$ axis. The propagation angle of the beam with respect to the $\zeta$ axis is $\Theta$, where $\tan \Theta \equiv \partial K_{\zeta} / \partial K_{\xi}=\partial_{V} K_{\zeta} / \partial_{V} K_{\xi}=V$ and $\partial_{V} \equiv \partial / \partial V$. 

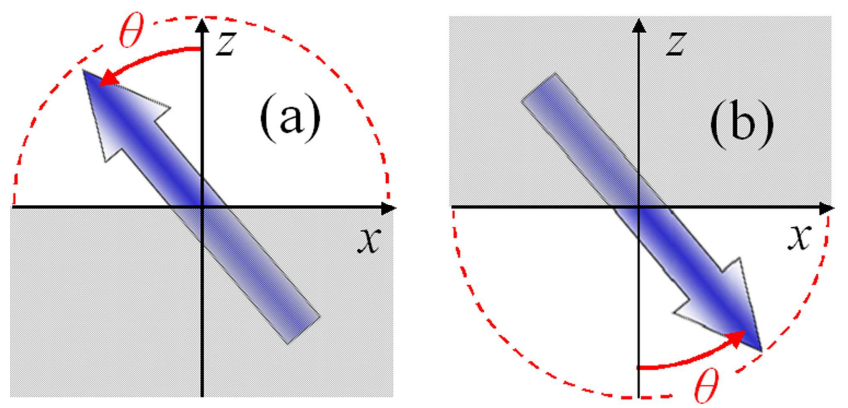

Fig. 2. (Color online) Propagation domains for (a) forward and (b) backward Helmholtz solitons. Each beam is restricted by the condition $-\infty \leq V \leq+\infty$, which corresponds to $-90^{\circ} \leq \theta \leq+90^{\circ}$ in the $(x, z)$ frame since $\tan \theta=(2 \kappa)^{1 / 2} V$. The grey region denotes forbidden directions. Since $x$ and $z$ are scaled by different factors $\left(L_{D} \gg w_{0}\right)$, the propagation angle $\theta$ in the $(x, z)$ frame is represented by the angle $\Theta$ in the $(\xi, \zeta)$ frame (see Fig. 1), where $\theta$ and $\Theta$ are related by $\tan \theta=(2 \kappa)^{1 / 2} \tan \Theta$. 

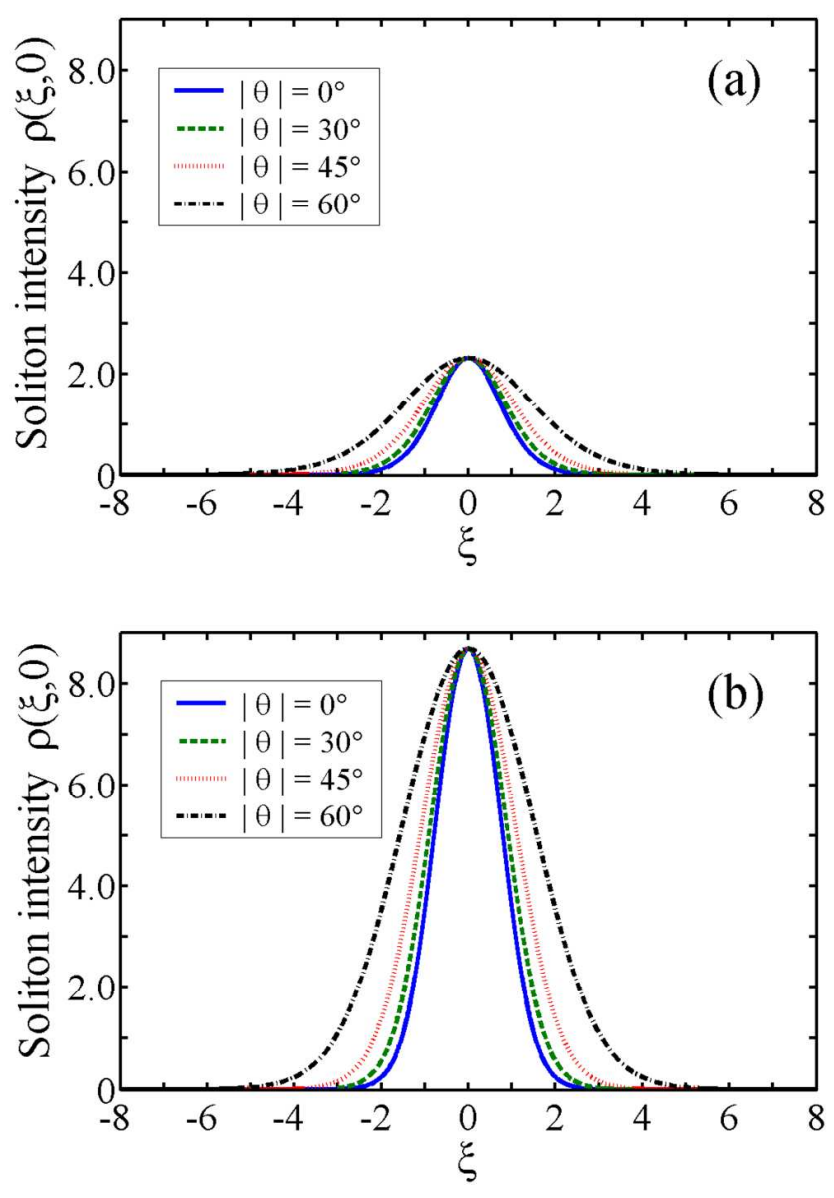

Fig. 3. (Color online) Angular beam-broadening effect for bistable Helmholtz solitons (9) when $\gamma=0.25$ for (a) lower- $\left(\rho_{0} \simeq 2.32\right)$ and (b) upper-branch $\left(\rho_{0} \simeq 8.68\right)$ solutions. Geometrical broadening is absent for the paraxial solution $\left(|\theta|=0^{\circ}\right)$. For a launching angle of $|\theta|=60^{\circ}$, the perceived width of the beam has doubled relative to the paraxial profile. 


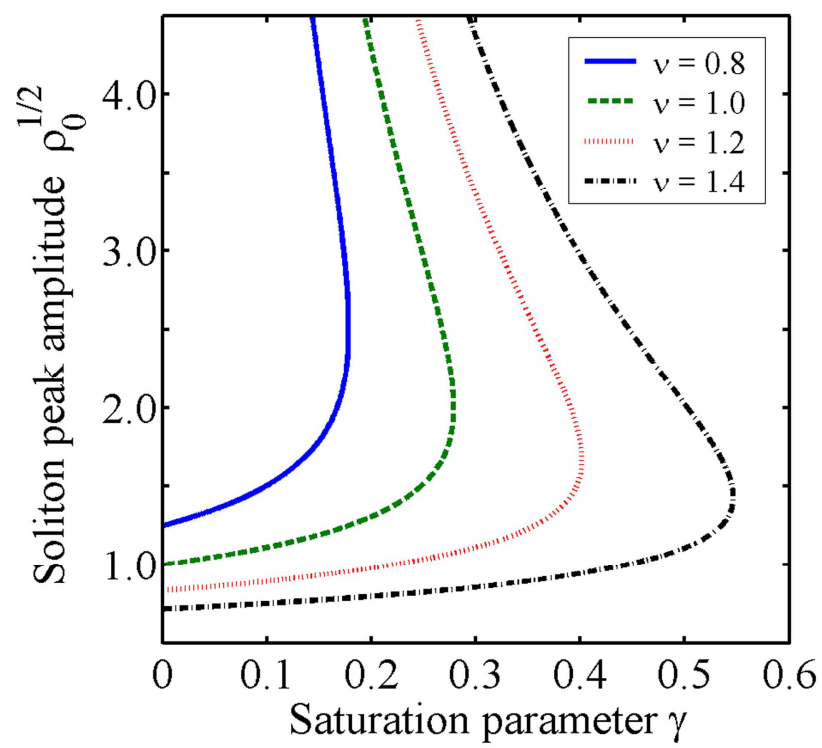

Fig. 4. (Color online) Curves defining non-degenerate bistable solution families for four different values of the width parameter $v$. These plots are obtained by solving Eq. (11) numerically. 


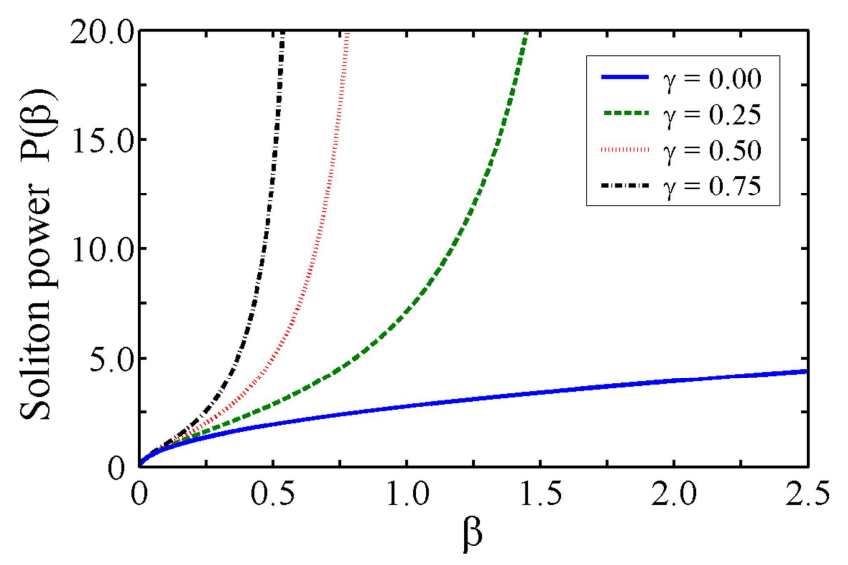

Fig. 5. (Color online) Paraxial beam power $P$ as a function of $\beta$ for four different values of the saturation parameter $\gamma$. The curve for $\gamma=0.00$ corresponds to a Kerr nonlinearity, where $\beta=\rho_{0} / 2$ [from Eq. (8)]. One finds that the slope $d P / d \beta$ is always positive (solitons predicted to be stable), and $P(\beta)$ is single-valued (no Kaplan-type degenerate bistability). 

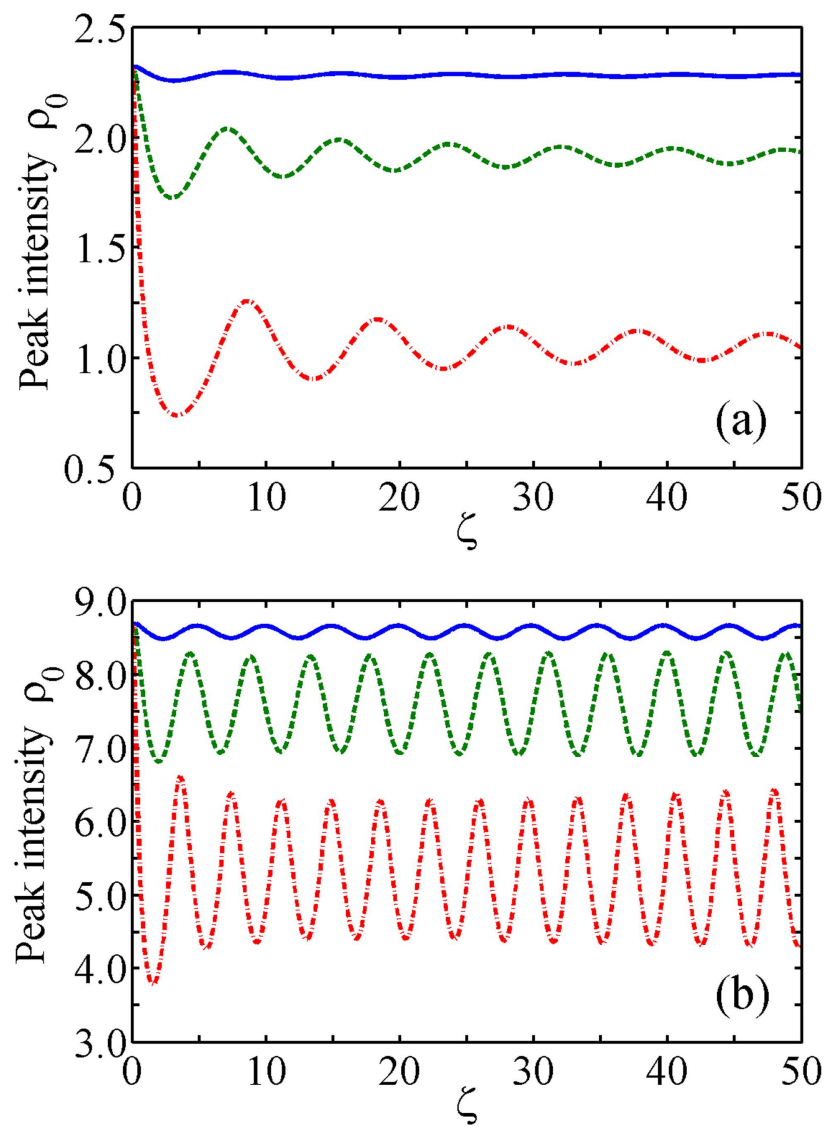

Fig. 6. (Color online) Beam reshaping simulations for (a) lower- and (b) upper-branch canonical solitons. Solid line: $|\theta|=10^{\circ}$, dashed line: $|\theta|=30^{\circ}$; dot-dashed line: $|\theta|=50^{\circ}$. Perturbed beams exhibit decaying oscillations in the amplitude, width, and area. These curves are universal, and hold for any combination of $\kappa V^{2}=\frac{1}{2} \tan ^{2} \theta$ [see text for the specific numerical values of $\kappa$ and $V$ used in these simulations]. 